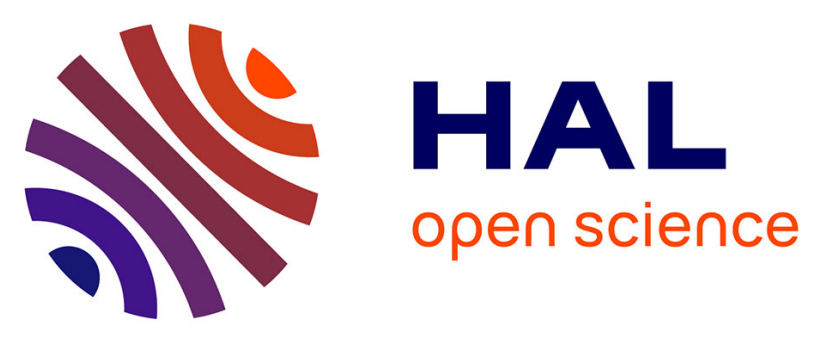

\title{
On the mixing enhancement in concentrated non-colloidal neutrally buoyant suspensions of rigid particles using helical coiled and chaotic twisted pipes:
}

A numerical investigation

T. Dbouk, C. Habchi

\section{To cite this version:}

T. Dbouk, C. Habchi. On the mixing enhancement in concentrated non-colloidal neutrally buoyant suspensions of rigid particles using helical coiled and chaotic twisted pipes: A numerical investigation. Chemical Engineering and Processing: Process Intensification, 2019, 141, pp.107540 -. 10.1016/j.cep.2019.107540 . hal-03485819

\section{HAL Id: hal-03485819 \\ https://hal.science/hal-03485819}

Submitted on 20 Dec 2021

HAL is a multi-disciplinary open access archive for the deposit and dissemination of scientific research documents, whether they are published or not. The documents may come from teaching and research institutions in France or abroad, or from public or private research centers.
L'archive ouverte pluridisciplinaire HAL, est destinée au dépôt et à la diffusion de documents scientifiques de niveau recherche, publiés ou non, émanant des établissements d'enseignement et de recherche français ou étrangers, des laboratoires publics ou privés.

\section{(c) (1) $\$$}

Distributed under a Creative Commons Attribution - NonCommerciall 4.0 International 


\title{
On the mixing enhancement in concentrated non-colloidal neutrally buoyant suspensions of rigid particles using helical coiled and chaotic twisted pipes: A numerical investigation
}

\author{
T. DBOUK ${ }^{\mathrm{a}, \mathrm{b}, *}$, C. HABCHI ${ }^{\mathrm{c}}$ \\ ${ }^{a}$ IMT Lille Douai, Energy Engineering Department, 59500 Douai, France \\ ${ }^{b}$ University of Lille, 59000 Lille, France \\ ${ }^{c}$ Notre Dame University - Louaize, Thermofluids Research Group, Zouk Mosbeh, Lebanon
}

\begin{abstract}
Nevertheless the huge amount of numerical and experimental works on noncolloidal suspension flows, most studies in the literature were limited to the dynamics in different geometries like in channels and pipes. To authors knowledge, mixing enhancement quantification and suspension mixing behavior in static mixers has been rarely studied before. In the present contribution, the mixing enhancement in isothermal monodisperse neutrally buoyant non-Brownian suspensions is investigated through transient 3D CFD simulations. It is quantified for suspension Reynolds $\left(R e_{S}\right)$ numbers varying between 50 and 150, for spheres diameters $\left(d_{p}\right)$ between 25 and $400 \mu m$ and for initial volume fractions of particles $(\phi)$ between 0.25 and 0.45 . The influences of $d_{p}, R e_{S}$ and $\phi$ on the overall mixing enhancement and on pressure drop are quantified for a suspension flow in a chaotic and helical separate pipes (static mixers of equal lengths). It is found that for all cases, the chaotic flow configuration has better mixing performances than the helical pipe flow with almost no significant increase in pressure losses. Moreover, good mixing quality is observed in both static mixers
\end{abstract}

\footnotetext{
* Corresponding author

Email address: talib.dbouk@imt-lille-douai.fr (T. DBOUK)
}

Preprint submitted to Chemical Engineering and Processing: Process Intensification

May 3, 2019 
(better at larger particles) where the mixing index ranges between 10 and $75 \%$ relative to the initial unmixed particles leaving the straight pipe.

Keywords: Suspensions mixing, non-colloidal concentrated suspensions, non-Brownian suspensions, chemical engineering process, rigid particles in liquid, chaotic advection, Dean cells, Computational Fluid Dynamics (CFD)

\section{Introduction}

Concentrated non-colloidal suspension flows of rigid particles are encountered in many industrial, pharmaceutical and petrochemical applications $[1,2$, 3]. For instance, the fabrication processes of many pharmaceutical products include concentrated rigid particles that are immersed in a liquid flow [4]. The homogeneity of the final product is of great importance and suitable mixers should be used and designed to achieve this good mixing quality. For that reason, understanding the mixing behavior and mixing efficiency in these complex flows of concentrated suspensions is a fundamental topic of research and development that has not been yet very well developed $[5,6]$.

In the present contribution, the mixing enhancement of concentrated noncolloidal suspensions of rigid particles by using helically coiled and chaotic twisted pipe flows (as static mixers) is investigated numerically through computational fluid dynamics (CFD). The numerical modeling approach used for this, is the one based on the Suspension Balance Model (SBM) that was developed and validated in previous works by $[7,8,9]$. In this paper, the mixing enhancement in isothermal monodisperse neutrally buoyant non-Brownian suspension flows is studied for effective suspension Reynolds numbers $R e_{S}$ between 50 and 150, for spherical particle diameters $\left(d_{p}\right)$ between 25 and 400 micrometers and for volume fractions $(\phi)$ varying between 0.25 and 0.45 . The suspensions are made of a Newtonian liquid that contains millions of immersed rigid spheres of 
phase-density ratio equal to unity $\left(\rho_{d}=\rho_{f}\right)$. The subscripts $d$ and $f$ represent the dispersed phase (particles) and the continuous-phase (liquid), respectively.

Understanding the particles transport and overall mixing behavior in such complex systems of concentrated suspensions is very important for creating better designs with higher mixing efficiency and thus improving the selectivity of the final products.

Experimentally speaking, over the years, researchers have been studying suspension flows to quantify the influence of: the local shear rate, particles diameter, particles polydispersity, micro-structure and bulk volume fraction $(\phi)$ on the shear-induced migration (SIM) $[10,11,12,13,7]$, the suspension effective viscosity $[14,15,8]$ and the normal stress differences (NSD) $[16,17,8]$. Experimental measurements using different techniques like nuclear magnetic resonance (NMR), particles image velocimetry (PIV) and enhanced rheometry, have been applied to quantify the SIM, the suspension micro-structure, the NSD, and the effective bulk flow behavior in different configurations [16, 18, 19, 20, 21]. Moreover, the influence of heat transfer on the SIM and the effect of buoyancy on suspension dynamics have been also studied in the literature [22, 9, 23].

Numerically speaking, scientists have been trying to quantify the complex rheology of laminar and Stokes flows of concentrated suspensions of rigid particles $[24,25]$. The flows of concentrated neutrally buoyant isothermal suspensions are known to exhibit a phenomenon of SIM in addition to a non-Newtonian behavior characterized by the presence of a first, and a second NSD $N_{1}$ and $N_{2}$, respectively [8]. In fact, modeling and simulation of concentrated suspensions is a very complex task due to the presence of a huge number of particles in the surrounding liquid or fluid. Thus, one must consider the particle-particle and fluid-particle interactions (for example, the hydrodynamic and contact forces). Lagrangian approaches can have several limitations such as in multiphyscial 
coupling in the presence of millions of particles in the flow. If millions of particles are present, the Lagrangian techniques can be computationally expensive (of high cost). Some examples on Lagrangian approaches are the Discrete Elements Method [21], Eulerian-lagrangian localized adjoint methods (ELLAM) [26], the Lattice Boltzmann Method (LBM) [27] and the Particles Dynamics Methods (PDM) [28]. When millions of particles are present in the suspension, the Eulerian approaches can be very beneficial over the Lagrangian ones in terms of computational cost. This is because they can represent a very large number of particles in the flow by applying averaging techniques. Several numerical studies have been conducted to quantify the potential of applying different numerical methods in predicting the SIM and the NSD in laminar concentrated non-Brownian suspensions [29]. In this contribution, the continuum macroscopic Eulerian approach based on the concentrated suspension model developed by $[7,8,9,23]$ is considered.

Nevertheless the huge amount of numerical and experimental works on suspension flows, most studies in the literature are limited to the dynamics of concentrated suspension flows in different geometrical configurations. However, to author's knowledge, mixing enhancement quantification and mixing behavior in concentrated non-colloidal suspension flows has been rarely studied before $[30,31,32]$.

Mixing and heat transfer enhancement in different geometries like raccoon, helically coiled and chaotic twisted pipe flows was extensively studied in the literature for Newtonian fluid flows [33, 34, 35, 36, 37], Non-Newtonian fluids [38] immiscible liquid/liquid dispersion [39, 40] and nanofluid flows [41, 42]. In a curved pipe, the centrifugal force induces a secondary flow consisting of two counter-rotating cells called Dean roll-cells [43, 44]. These cells, are superimposed on the axial flow and play an important role in mixing and heat transfer 
enhancement. In the chaotic flow configuration, the $90 \mathrm{deg}$ bend is rotated in the orthogonal plane with respect to the preceding one. This configuration breaks the symmetry of the helical pipe flow and generates chaotic advection [45]. Thus can further enhance the transport phenomena relative to helical pipe flow with almost no increase in pressure losses, especially at relatively low Reynolds numbers. This is in contrast to using vortex generators which enhances the mixing process at the penality of increasing the power consumption [46, 47]. Owing to the chaotic advection, the fluid particles will have higher chance to visit regions of higher shear and elongation rates. Moreover, the fluid particles in helical pipe stay trapped inside the Dean roll cells, which is not the case in chaotic twisted pipe flow. For instance, Mokrani et al. [33] show that the heat transfer in chaotic twisted pipe is enhanced by about 13 to $28 \%$ relative to helical coiled pipe. Moreover, Habchi et al. [39] show that the homogeneity of immiscible liqui/liquid dispersion is enhanced by about $40 \%$ when using chaotic pipe relative to helical pipe flow with almost no increase in pressure losses.

In the present contribution, the mixing enhancement in isothermal monodisperse neutrally buoyant non-Brownian suspension flows is investigated numerically. To the authors knowledge, this is addressed for the first time in the literature. The influence of the particles diameter, the suspension effective Reynolds number and the particles volume fraction on the overall mixing enhancement is quantified by applying both a chaotic and a helical pipe (as static mixers each of circular cross section). Results are presented, discussed and analyzed after comparisons with the results for flow of same suspensions but in a straight pipe. 


\section{Problem Description and Numerical Method}

\subsection{Background}

If a homogeneous suspension of neutrally buoyant rigid spheres in a liquid is put into a straight pipe flow, a phenomenon of SIM of particles will occur such that the particles migrate from zones near the walls toward the center-line of the pipe $[12,48]$. This SIM phenomenon, as illustrated in figure 1 , results in a nonhomogeneous particles distribution at the outlet which is inconvenient for many pharmaceutical and engineering processes. The SIM depends mainly on the suspension flow effective properties (inertial, viscosity, etc.), the flow geometry (type, dimensions, etc.) and the immersed particles nature (size, roughness, polydispersity, etc.).

For the last two decades, experimental and numerical studies on SIM were conducted in order to better understand this phenomenon and to quantify it in several geometries and flow regimes [49]. Nevertheless, the reverse problem have been never tackled in the literature, i.e. the mixing process where we go from SIM heterogeneity to particles homogeneity. The SIM mechanisms behind the SIM can be seen as shear rate gradients which induce gradients in the particle stress that result in concentration gradients in the suspension.

In this section, starting from a homogeneous particles distribution (or uniform local volume fraction of particles), the SIM is first quantified and recovered at the outlet of a long straight pipe at the steady state for concentrated suspension flow. Then the resulting non-homogeneous particles distribution at the outlet of the straight pipe (with its corresponding effective velocity field) are then injected at two different inlets corresponding to the helical and chaotic pipes. The objective is to enhance the homogeneity at the outlet of these two mixers. 


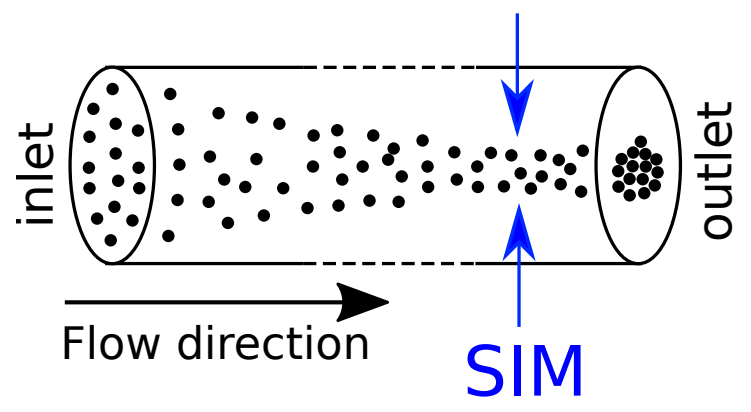

Figure 1: Shear-induced migration of spherical particles in a concentrated neutrally buoyant $\left(\rho_{\text {particles }}=\rho_{\text {fluid }}\right)$ suspension flow inside a pipe of circular cross section.

\subsection{Computational domain}

Three computational domains are used in the present study: straight pipe, helical pipe and chaotic twisted pipe (all of same diameter $D=4 \mathrm{~mm}$ ) as shown in figure 2. This diameter was taken so that to have a large number of particles per pipe's diameter varying between 10 and 160. This is important to represent the continuum medium of the concentrated suspension of particles. This diameter is also of the same order as used by Habchi et al. 2009 [39, 40] to study liquid-liquid dispersion. The straight pipe length is varying and each time in order to obtain a fully developed regimes. This is estimated for each $\phi$ and Re values as it will be explained in Section 2.3. However, the helical and chaotic mixers are of same length corresponding to 7 twisted $90^{\circ}$ elbows. This number of elbows ( $i 5)$ was chosen based on the pipe's length estimation for a fully developed state of suspension flow as it will be explained next, and due to previous works on liquid-liquid mixing enhancement through advection in chaotic pipes $[40,46,50]$.

\subsection{Straight pipe length estimation for a fully developed state of suspension flow}

When a homogeneous concentrated neutrally buoyant $\left(\rho_{p}=\rho_{f}\right)$ non-colloidal suspension is injected in a straight pipe from inlet to outlet (see figure 1), SIM occurs resulting in a developing non-homogeneous concentration profile at the 


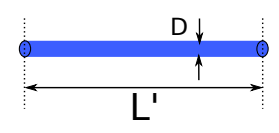

(a) Straight

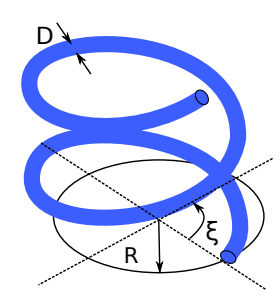

(b) Helicoidal

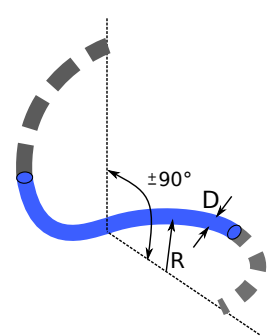

(c) Chaotic twisted

Figure 2: Three different pipe configurations. (a) straight; (b) helical pipe; (c) chaotic twisted pipe. $R=45 \mathrm{~mm} ; \xi / D \approx 0.16 ; D=4 \mathrm{~mm} ; L^{\prime} / D \approx 121$. $\xi$ is the pitch of the helical pipe, and $R$ its radius of curvature.

outlet at the steady state. For that reason, in order to insure a fully developed state for the concentrated suspension flow, one should estimate the minimum straight pipe length $\left(L^{\prime}\right)$ required (used in our computational domain) as illustrated in figure 3 .

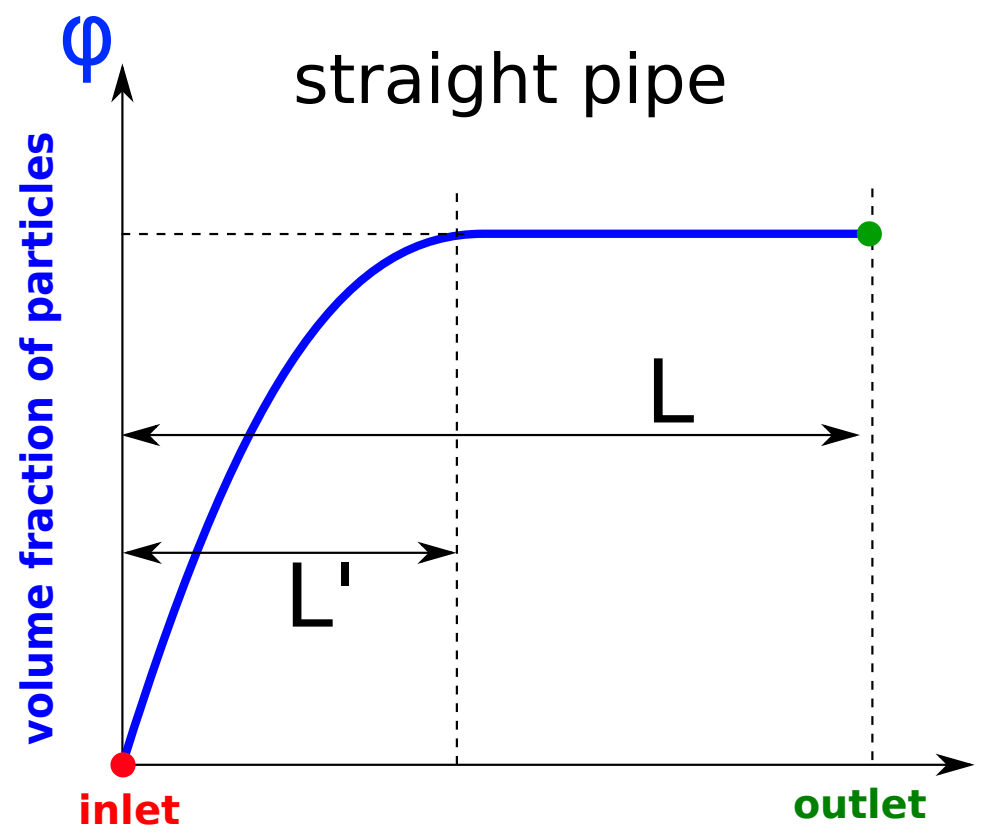

Figure 3: Fully developed state of a concentrated neutrally buoyant suspension flow in a straight pipe of circular cross section. $L^{\prime}$ is the estimated minimum pipe's length required. Volume fraction along the suspension flow direction is at the center-line. 
Nott and Brady (1994) [51] showed that profiles are fully developed if:

$$
\left(\frac{L^{\prime}}{2 D}\right) \geq \frac{1}{12 g(\phi)}\left(\frac{D}{d_{p}}\right)^{2}
$$

where $g(\phi)$ stands for the dependence of the shear-induced diffusion coefficient on the bulk particle concentration $(\phi)$. After a good fitting to their experimentally measured diffusion coefficients, Leighton and Acrivos (1987) [10, 11] and later Chapman (1990) [13] found that $g(\phi)$ is best described as the following:

$$
g(\phi)=\frac{1}{3} \phi^{2}\left(1+0.5 e^{8.8 \phi}\right)
$$

The effective Reynolds number $\left(R e_{S}\right)$ of the suspension is defined in the present work based on the uniform velocity value imposed at the inlet of the straight pipe as the following:

$$
\operatorname{Re}_{S}=\frac{U_{i n} D}{\eta_{S}}
$$

$R e_{S}$ is related linearly to the suspension Dean number such that:

$$
D e_{S}=R e_{S} \sqrt{\frac{D}{2 R}}=0.21 R e_{S}
$$

$R$ is the radius of curvature of the helical pipe, $D$ the pipe's diameter and $U_{i n}$ the uniform velocity value imposed at the inlet of the straight pipe. $\eta_{S}$ is the suspension effective kinematic viscosity (particles' diameter independent) that can be measured experimentally by Rheology instruments and then can be fitted as function of local volume fraction $(\phi)$ and the local shear rate $(\dot{\gamma})$ (see Rebouças et al. 2016 [52]) as a generalized Cross model [53] as the following:

$$
\eta_{S}(\dot{\gamma}, \phi)=\eta_{\infty}(\phi)+\frac{\eta_{0}(\phi)-\eta_{\infty}(\phi)}{1+b P e_{a}}
$$


$b$ is a rheological fitting parameter $(\approx 5.7$ for hard spheres $[53,52])$ and $P e_{a}$ is the particle Péclet number given by:

$$
P e_{a}=\eta_{f} \dot{\gamma} a^{3} / \kappa_{B} T
$$

that usually tends to infinite values in the case of non-colloidal suspensions. $\kappa_{B}$ is the Boltzmann's constant, $T$ absolute temperature, $a$ particle radius and $\dot{\gamma}$ the local shear rate defined as function of the local strain rate tensor $\mathbf{E}$ as the following:

$$
\dot{\gamma}=\sqrt{2 \mathbf{E}: \mathbf{E}} ; \quad \mathbf{E}=0.5\left(\nabla \mathbf{U}+\nabla \mathbf{U}^{\mathbf{T}}\right)
$$

Many constitutive equations have been developed in the literature such as those by Maron and Pierce (1956) [54] and Krieger and Dougherty (1959) [14] that have the following form:

$$
\begin{gathered}
\eta_{0}(\phi)=\eta_{f}\left(1-\frac{\phi}{\phi_{\max -0}}\right)^{-\alpha_{0}} \\
\eta_{\infty}(\phi)=\eta_{f}\left(1-\frac{\phi}{\phi_{\max -\infty}}\right)^{-\alpha_{\infty}}
\end{gathered}
$$

$\phi_{\max }$ corresponds to maximum packing volume fraction of particles that depends on the particles form and their surface roughness. $\alpha$ is a fitting parameter that can vary between 1.67 and 2 for rigid spheres as observed experimentally in the literature (i.e. see $[52,55])$. The subscripts 0 and $\infty$ corresponds to low and high shear rate $(\dot{\gamma})$ local regimes, respectively.

An illustrative example for the behavior of different constitutive relations of suspension viscosity can be seen in figure 4 (for more details see [55]). The suspension relative viscosity will reach infinity value as the volume fraction $(\phi)$ 


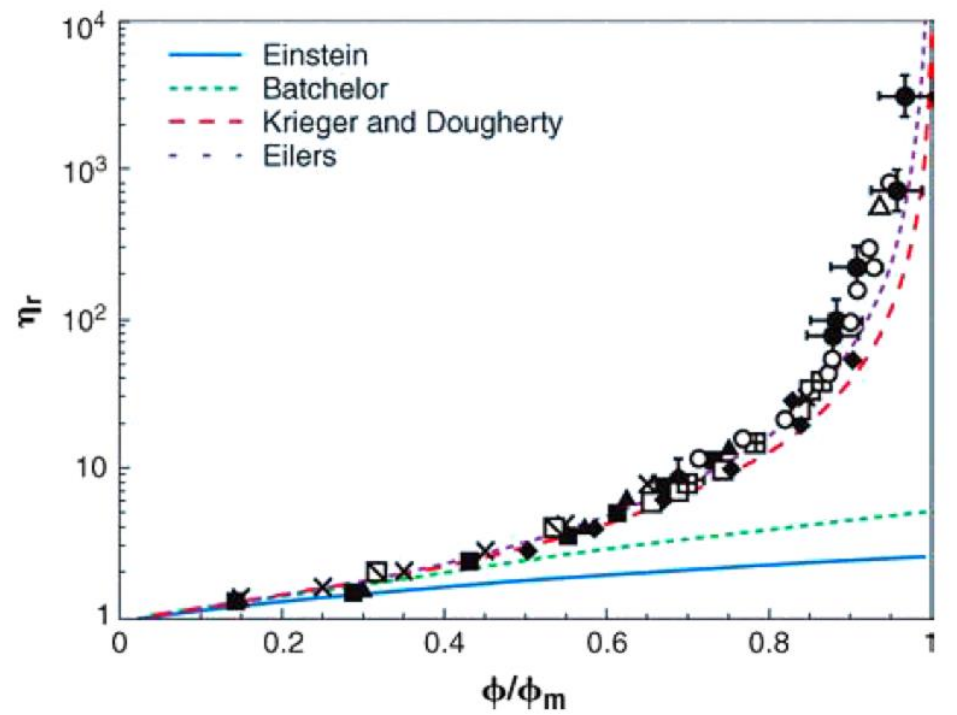

Figure 4: From (Stickel and Powell 2005 [55]): Effective suspension viscosity $\eta_{S}$ as a function of the normalized volume fraction $\left(\phi / \phi_{\max }\right)$. The symbols correspond to data presented in Chang and Powell 1994 [56].

approaches $\phi_{\max }$. Usually for rigid spherical beads as known form experimental rheology measurements from the literature [55], $\phi_{\max }$ vary between $\phi_{\max -0}=$ 0.58 and $\phi_{\max -\infty}=0.68$ which were both considered in the present modeling (representing both maximum packing values respectively at both low and high local shear rates of the suspension [52]).

In the present study, the condition in equation (1) is verified for each case so that to ensure fully developed concentration profile at the straight pipe outlet.

\subsection{Boundary and operating conditions}

The boundary conditions are illustrated in figure 5 . The velocity and concentration fields collected at the outlet of the straight pipe are injected to the helical and chaotic mixers. No-slip boundary condition is imposed on the pipe wall and fixed pressure at the mixers outlets.

The operating conditions applied in the present CFD simulations are shown in Table 1. 


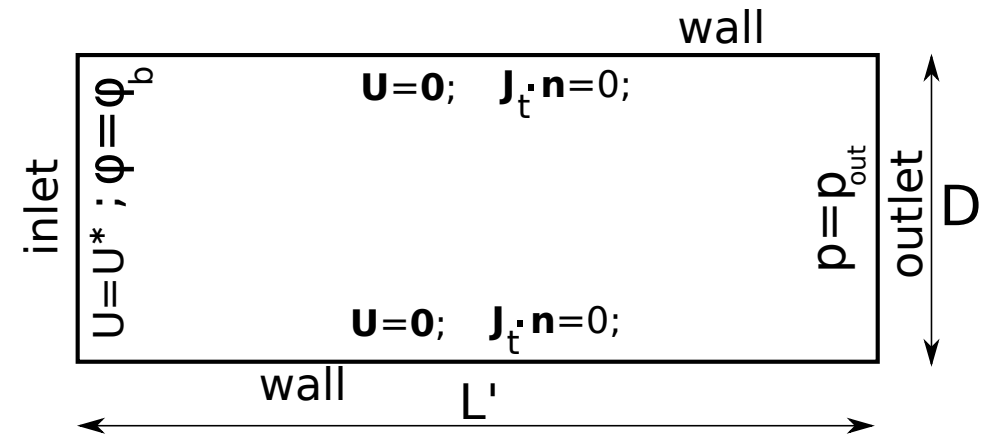

Figure 5: The boundary conditions applied in all of the present CFD simulations. $U^{*}($ straight pipe $)=U_{\text {in }}$ (imposed value at inlet $) ; U^{*}($ chaotic pipe $)=$ $U_{\text {out }}($ straight pipe computed at steady state $) ; U^{*}($ helicoidal pipe $)=$ $U_{\text {out }}$ (straight pipe computed at steady state)

\begin{tabular}{|c|c|c|c|}
\hline $\mathrm{Re}^{\phi}$ & 0.25 & 0.35 & 0.45 \\
\hline 50 & 100 & 100 & 100 \\
\hline 100 & 100 & $25 ; 60 ; 100 ; 400$ & 100 \\
\hline 150 & 100 & 100 & 100 \\
\hline
\end{tabular}

Table 1: Operating conditions in the three geometries. The values in each interior cell correspond to the particles diameter (in $\mu m$ ) at given $R e$ and $\phi$.

\subsection{Governing equations and numerical method}

Considering incompressible isothermal $(T=293 K)$ non-Brownian neutrally buoyant concentrated suspensions of rigid spheres immersed in a Newtonian fluid, the suspension flow governing equations following the suspension balance model (SBM) of $[7,22]$ are given as the following:

$$
\begin{gathered}
\nabla \cdot \mathbf{U}=\mathbf{0} \\
\frac{\partial \mathbf{U}}{\partial t}+\nabla \cdot(\mathbf{U} \otimes \mathbf{U})-\nabla \cdot \boldsymbol{\Sigma}=0 \\
\frac{\partial \phi}{\partial t}+\mathbf{U} \cdot \nabla \phi+\nabla \cdot \mathbf{J}_{\mathbf{s}}=\mathbf{0}
\end{gathered}
$$


Where $t$ denotes the time, $\mathbf{U}$ is the averaged suspension velocity, $\phi$ the local volume fraction of particles and the suspension overall stress tensor $\boldsymbol{\Sigma}$ is defined as $\left(\boldsymbol{\Sigma}=\boldsymbol{\Sigma}_{f}+\boldsymbol{\Sigma}_{p}\right)$. For more details about both $\boldsymbol{\Sigma}_{f}$ and $\boldsymbol{\Sigma}_{p}$, such as their mathematical descriptions and derivations, the reader may refer to the recent works of $[7,8,9]$.

The volume fraction $\phi$ is related to the particles number density $n$ such that $n=\frac{3 \phi}{4 \pi\left(\frac{d_{p}}{2}\right)^{3}} V$ with $V$ being the total volume of the bulk suspension in the domain of interest.

$\mathbf{J}_{\mathbf{s}}$ represents the SBM migration flux [7] written as follows:

$$
\mathbf{J}_{s}=\frac{d_{p}^{2}}{18 \eta_{f}} f(\phi) \nabla \cdot \boldsymbol{\Sigma}_{p}
$$

In this equation, $f(\phi)$ is the sedimentation hindrance function $f(\phi)$ which is given by:

$$
f(\phi)=\left(\frac{1-\phi}{\phi_{\max }}\right)(1-\phi)^{\alpha-1} ; \alpha \in[2,4]
$$

The form of $f(\phi)$ in equation (14) is proposed by $[15,57]$ as a sedimentation hindrance function that represents the mobility of the particle phase.

The Finite Volume Method (FVM) $[58,59]$ is then applied as the numerical technique to discretize the system of partial differential equations that were all implemented and solved iteratively in the open source CFD platform OpenFOAM [60]. To solve efficiently for the coupling of the pressure-velocity fields, the PIMPLE algorithm [58, 59] was applied which permits for dynamic time step while respecting the $C F L<C F L_{\max }$ condition (here $C F L_{\max }$ was set to 0.5$)$. This is essential to reduce the overall computational time in transient simulations while retaining a good convergence with good precision of results. 


\subsection{Meshing and mesh sensitivity analysis}

A 3D mesh sensitivity analysis was conducted for the most critical conditions $\left(R e=150, \phi_{b}=0.45, d_{p}=100 \mu m, \rho_{p}=\rho_{f}\right)$ of the suspension flow in a straight pipe in order to quantify the minimum base cell size required for all the present CFD simulations. Polyhedral finite volume cells of total number $N_{\text {cells }}$ varying between $10^{5}$ and $2 \cdot 10^{5}$ have been tested respecting always the $C F L$ and numerical diffusion conditions.

An example of the two 3D meshes applied can be found in figures 6 and 7 .

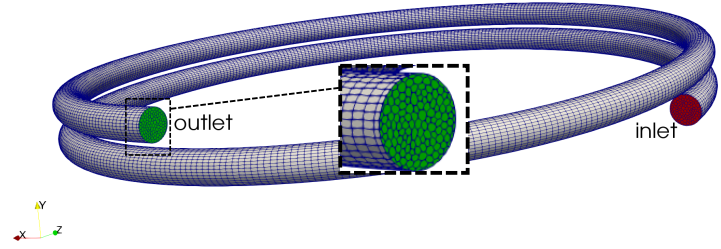

Figure 6: Three-dimensional mesh applied in the Helical pipe geometry.

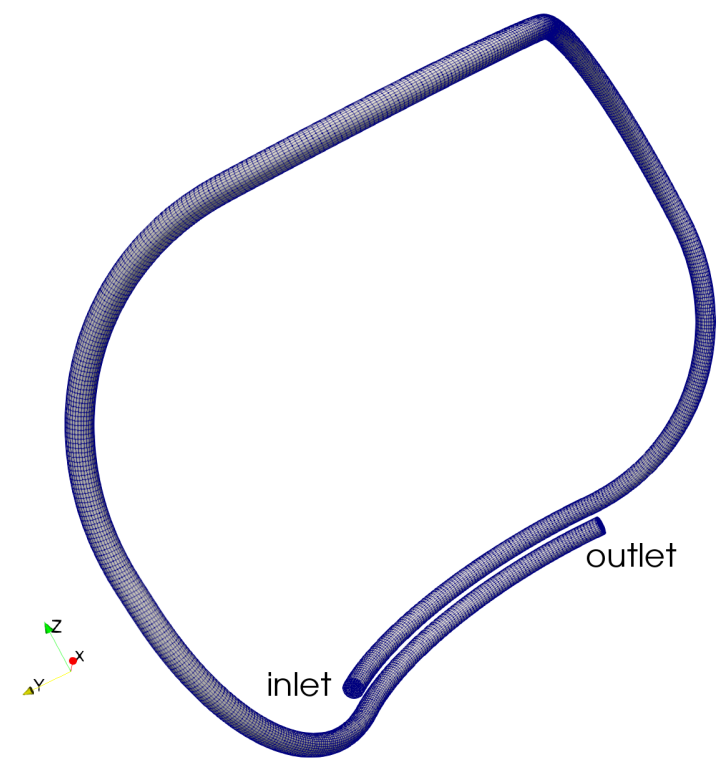

Figure 7: Three-dimensional mesh applied in the Chaotic pipe geometry.

The parameters we considered for the mesh sensitivity analysis are local 


\begin{tabular}{lccc}
\hline & Mesh 1 & Mesh 2 & Mesh 3 \\
\hline Cell number & $1 \times 10^{5}$ & $1.5 \times 10^{5}$ & $2 \times 10^{5}$ \\
\hline$\phi_{\max }$ & 0.5316 & 0.5148 & 0.5153 \\
\hline$O r_{\phi_{\max }}$ & & & 8.1 \\
\hline$G C I_{\phi_{\max }}$ & & & $0.24 \%$ \\
\hline$U_{\max }(\mathrm{m} / \mathrm{s})$ & 1.1262 & 1.2528 & 1.2475 \\
\hline$O r_{U_{\max }}$ & & & 7.3 \\
\hline$G C I_{U_{\max }}$ & & & $1.03 \%$ \\
\hline
\end{tabular}

Table 2: Mesh sensitivity analysis.

values of $\phi$ and $U$. For instance, here we present the mesh sensitivity in a location with the highest discretization error occurring at the outlet of the computational domain. The mesh sensitivity analysis is performed according to the procedure proposed by Celik et al. [61] where the grid convergence index (GCI) and order of accuracy $(\mathrm{Or})$ are evaluated from at least three different mesh densities. As shown by GCI values in Table 2, the highest discretization error did not exceed $0.24 \%$ for $\phi_{\max }$, while it did not exceed $1.03 \%$ for $U_{\max }$.

\section{Results and discussions}

\subsection{Effect of Dean roll cells on concentration distribution}

As discussed in the introduction section, Dean roll cells are generated in curved pipe flows due to centrifugal force. These cells are well observed in the present study as shown in figure 8 for the operating conditions: $R e=100$, $d_{p}=400 \mu m$ and $\phi_{b}=0.35$, and figure 9 for the operating conditions: $R e=$ $100, d_{p}=100 \mu m$ and $\phi_{b}=0.35$. These roll cells play the role of internal agitator to the flow and enhance the radial mixing processes. As shown in this figure, the SBM is well obtained in the straight pipe flow where the particles converge towards the flow core in the center-line. The helix and chaotic flow configurations are then used to homogenize the concentration profile thanks to the Dean roll cells as can be seen in the volume fraction results in figure 8 for the 
operating conditions: $R e=100, d_{p}=400 \mu m$ and $\phi_{b}=0.35$. The effect of the streamwise vortices on the concentration profile is well depicted in this figure especially for the classical helical configuration where high concentration values are pushed by the common flow towards the edge and low concentration values are being pushed from the near wall region towards the flow core. Meanwhile, a slight asymmetry is obtained in the chaotic flow configuration due to the periodic alternation of the curved pipes orientation. This asymmetry brakes the concentration profile and further enhances the mixing process as will be discussed in the next sections.

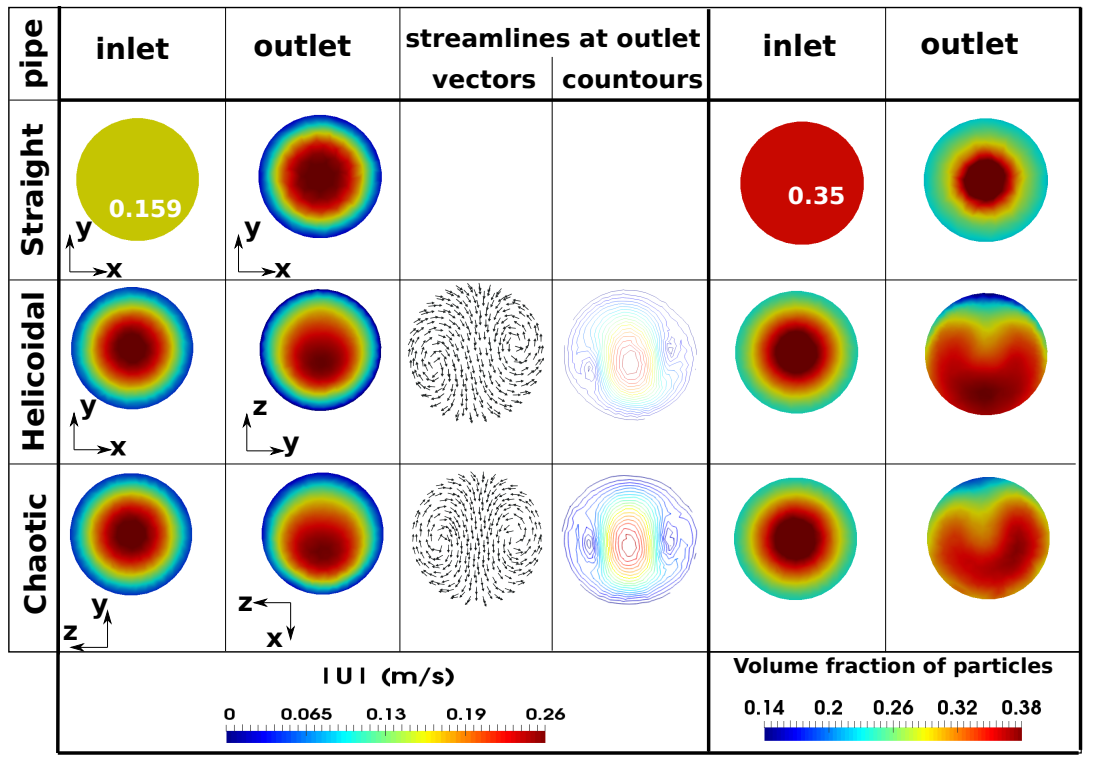

Figure 8: From left to right: Velocity magnitude, streamlines, velocity contours and volume fraction of particles for straight, helicoidal and chaotic pipes. Results at steady state for the operating conditions: $R e=100, \mathbf{d}_{\mathbf{p}}=\mathbf{4 0 0} \mu \mathbf{m}$ and $\phi_{b}=0.35$.

Figures 10 and 11 illustrate the evolution of the volume fraction of particles distribution at different elbows exits cross-section for the operating conditions: $\operatorname{Re}=100, d_{p}=25 ; 400 \mu m$ and $\phi_{b}=0.35$. It can be observed that good mixing begins to occur starting at the exit cross-section of the $3^{\text {rd }}$ elbow. At particles diameter of $d_{p}=25 \mu \mathrm{m}$ lower volume fractions range is observed than in the 


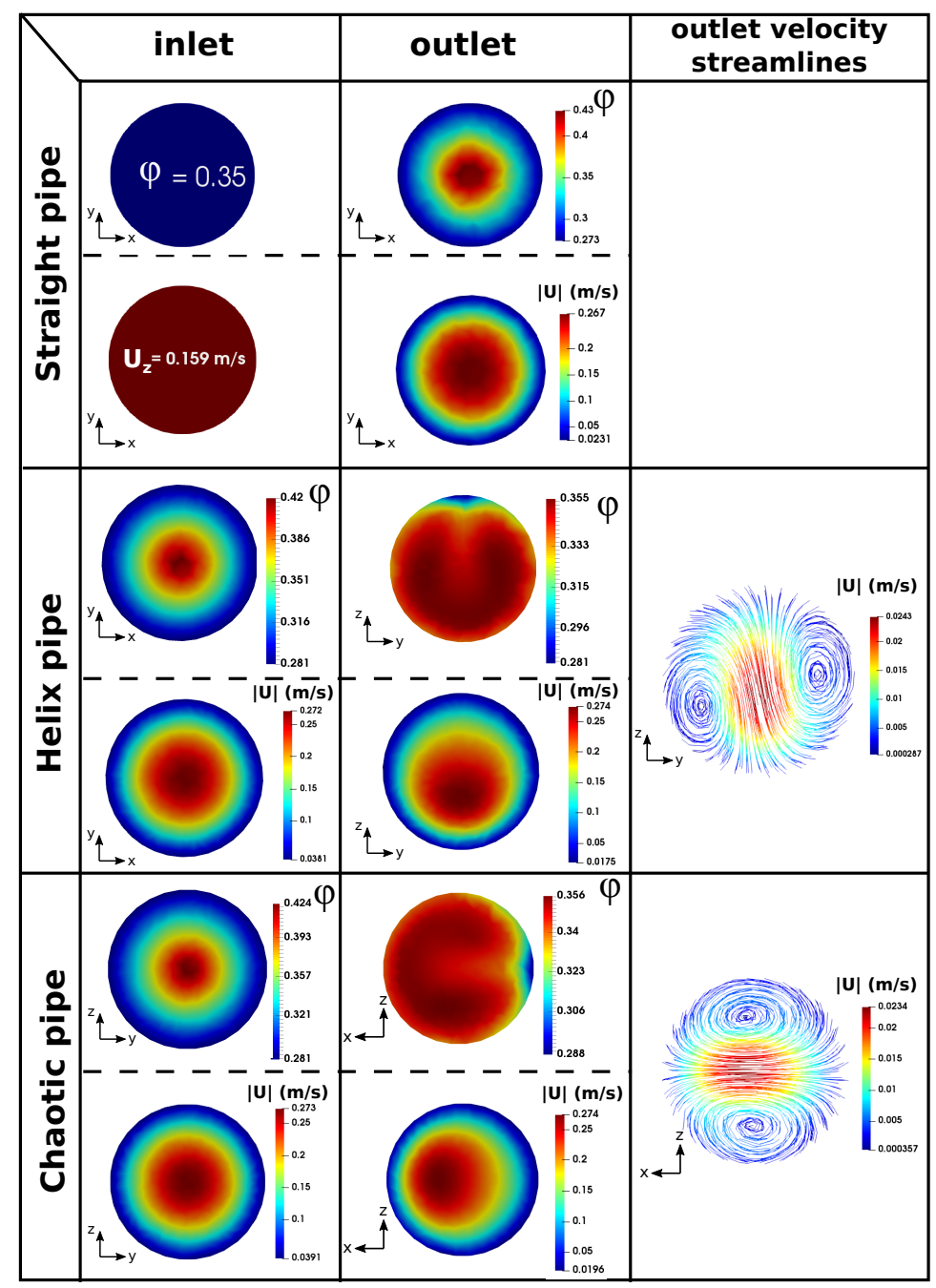

Figure 9: Velocity magnitude, volume fraction and velocity streamlines at the inlet and outlet of: straight, helicoidal and chaotic pipes. Results at steady state for the operating conditions: $R e=100, \mathbf{d}_{\mathbf{p}}=\mathbf{1 0 0} \mu \mathbf{m}$ and $\phi_{b}=0.35$.

case of particles diameter of $d_{p}=400 \mu \mathrm{m}$. This is due to the fact that the injected concentration profiles at inlet correspond well to the same ranges, since SIM was more manifested at $d_{p}=400 \mu \mathrm{m}$ rather than at $d_{p}=25 \mu \mathrm{m}$.

Figure 12, and figure 13 illustrate the evolution of the volume fraction of particles distribution at different elbows exits cross-section for the operating 


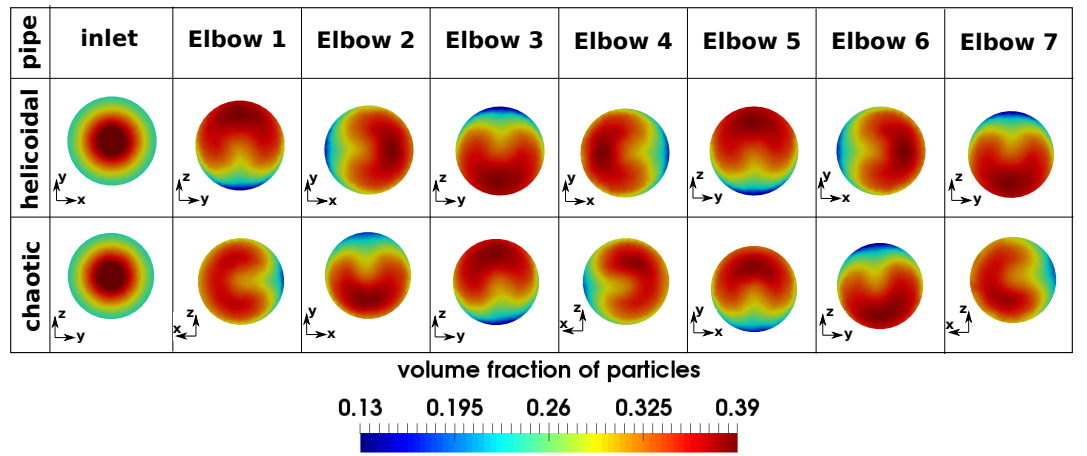

Figure 10: Volume fraction of particles at the exits of the $790^{\circ}$ elbows: helical and chaotic pipes. Results at steady state for the operating conditions: $R e=100, d_{p}=400 \mu \mathrm{m}$ and $\phi_{b}=0.35$.

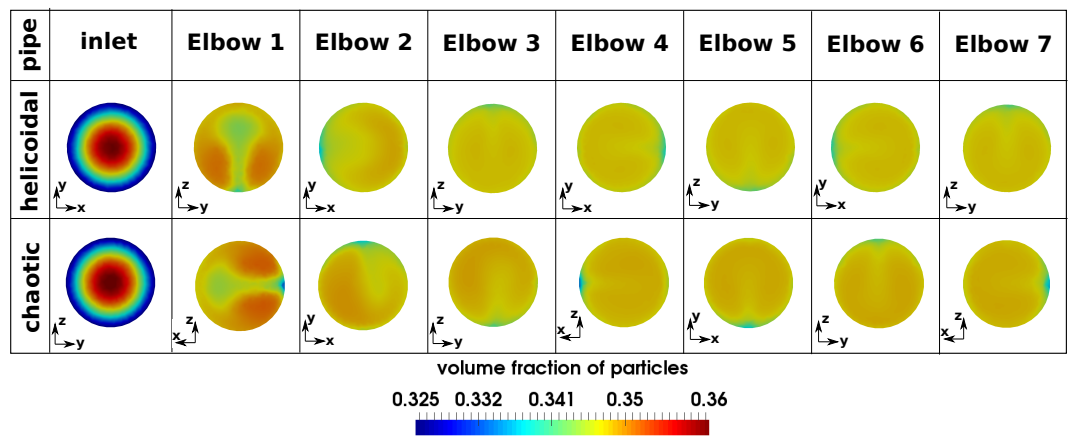

Figure 11: Volume fraction of particles at the exits of the $790^{\circ}$ elbows: helical and chaotic pipes. Results at steady state for the operating conditions: $R e=100, d_{p}=25 \mu \mathrm{m}$ and $\phi_{b}=0.35$.

conditions: $R e=100, d_{p}=100 \mu m$ and $\phi_{b}=0.35$ for the helical and chaotic pipes, respectively.

It can be clearly observed that good mixing is obtained starting form the exit of the $3^{r d}$ elbow which then retains a steady cross-sectional distribution of particles between the exits of elbows number 4 and 7 . 


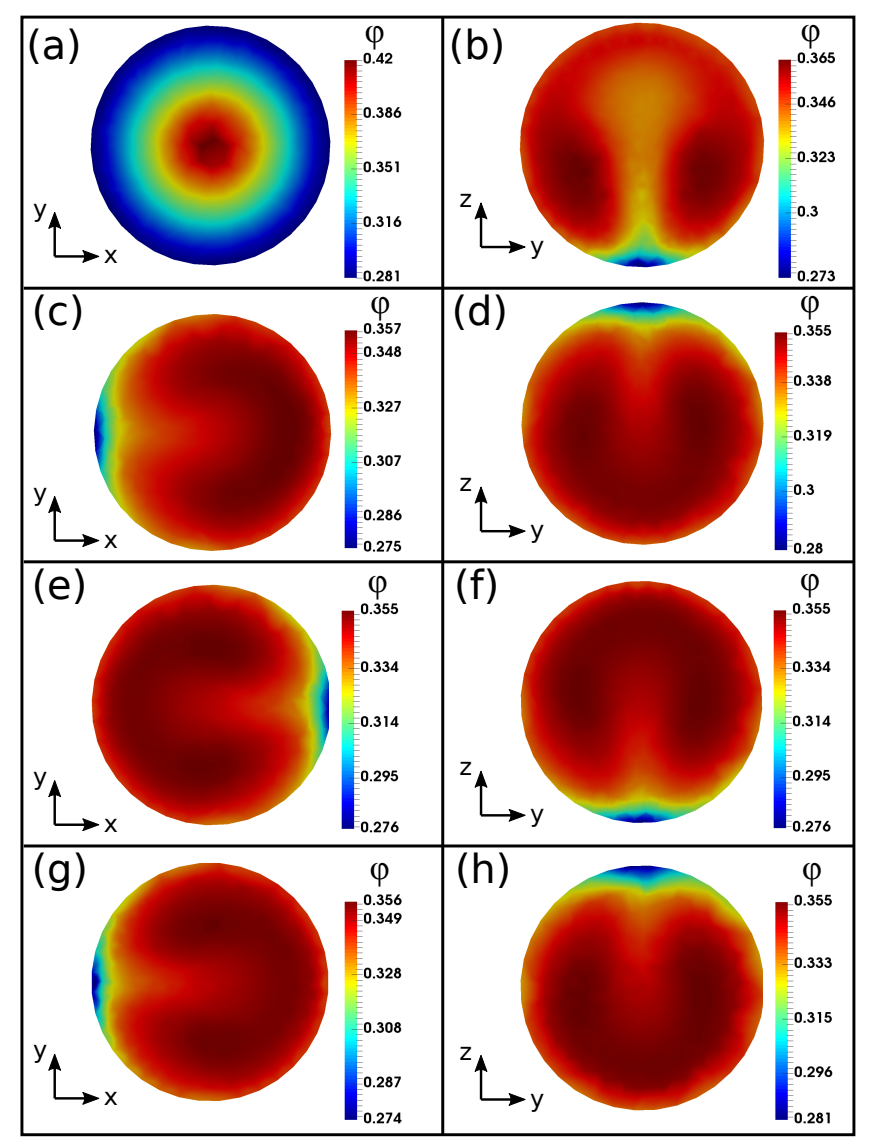

Figure 12: Volume fraction of particles at the exits of the $790^{\circ}$ elbows: helical pipe. Results at steady state for the operating conditions: $R e=100, d_{p}=100 \mu \mathrm{m}$ and $\phi_{b}=0.35$. (a): inlet; (b) to (h): elbow 1 to elbow 7 , respectively.

\subsection{Mixing index and friction factor}

To quantify the mixing process, we adopt the mixing index $M I$ based on the coefficient of variation of the particles concentration as follows:

$$
M I=1-\frac{C o V_{\text {outlet }}}{C o V_{\text {inlet }}}
$$

where the coefficient of variation $\mathrm{CoV}$ is obtained as follows:

$$
C o V=\frac{\sigma_{\phi}}{\hat{\phi}_{b}}
$$




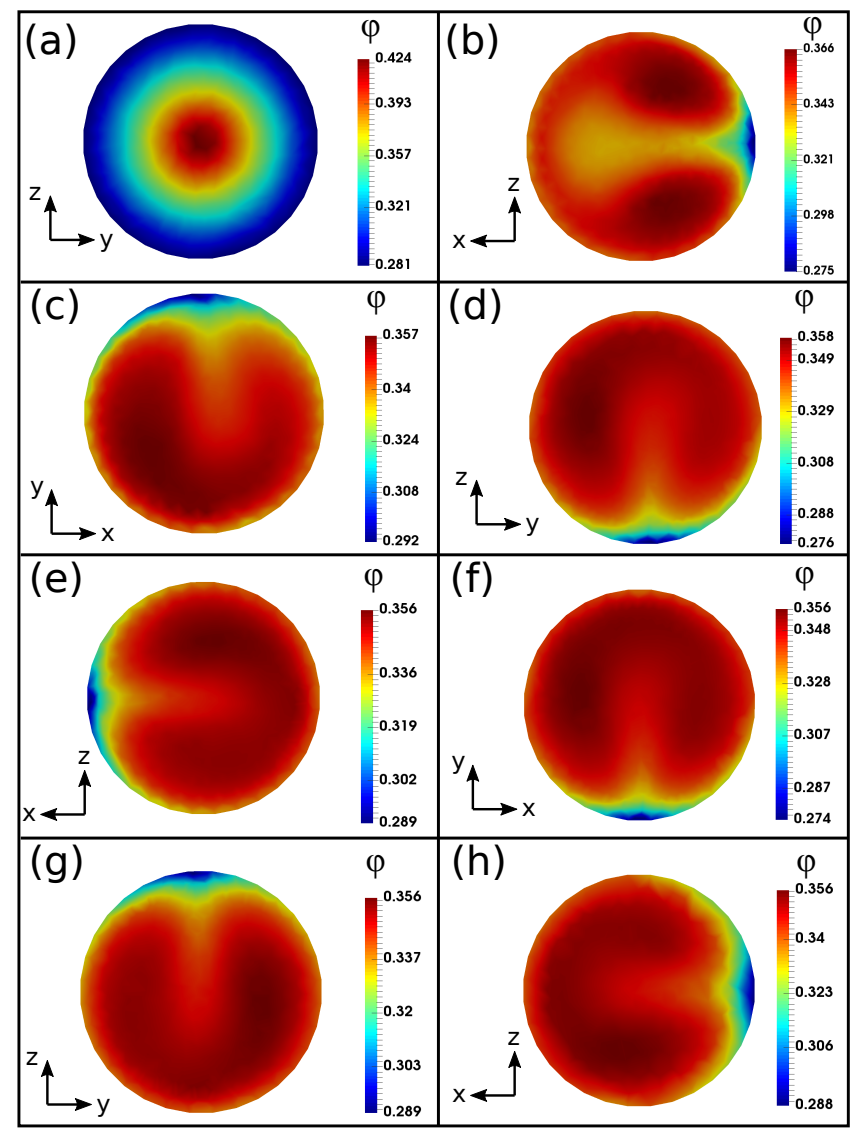

Figure 13: Volume fraction of particles at the exits of the $790^{\circ}$ elbows: chaotic pipe. Results at steady state for the operating conditions: $R e=100, d_{p}=100 \mu \mathrm{m}$ and $\phi_{b}=0.35$. (a): inlet; (b) to (h): elbow 1 to elbow 7 , respectively.

$\sigma_{\phi}$ is the standard deviation of the volume fraction at outlet with respect to the mean value $\hat{\phi_{b}}$. The mixing index is represented in $\%$ and it is directly related to the degree of homogeneity of the particles which is quantified by the $\mathrm{CoV}$. Good mixing is reflected by high values of $M I$, and vice-versa.

Meanwhile, good mixing could be accompanied by an increase in pressure drop. Thus the pumping power factor $F$ is used to quantify the possible increase 
in pressure losses and it reads $[62,50]$ :

$$
F=f R e_{S}{ }^{3}
$$

where $f$ is the friction coefficient obtained from the pressure difference between flow outlet and inlet $\Delta p$ as follows:

$$
f=\frac{2 D \Delta p}{\rho U_{i n}{ }^{2} L^{\prime}}
$$

\subsection{Effect of Reynolds number}

In this section we discuss variation of the mixing index and friction losses with the Reynolds number defined previously in equation (3). The variation of $M I$ versus $R e$ is presented in figure 14 for the different mean concentrations and for the two studied mixers, i.e. helical and chaotic pipe flows. First of all, the chaotic mixer shows better performances than the helical pipe configuration for all cases where the mixing index is around $5 \%$ higher. At $\phi_{b}=0.25, M I$ first increases in both configurations from the lowest Re to the intermediate and then decreases. This parabolic behavior at the lowest $\phi_{b}$ is directly related to the way the particles are interacting between themselves and with the fluid flow. At $\phi_{b}=0.35$ and $0.45 M I$ shows a decrease with increasing Re which is more pronounced at the higher $\phi_{b}$ case. In fact, at low $R e$, the residence time is larger than high Re cases. Thus the solid particles have more time to mix and thus better $M I$ is obtained at the low $R e$ values. This observation contradicts the behavior of liquid mixing applications in which the mixing performance increases with increasing $R e$ due to the increase in the flow momentum [39]. However, in presence of solid particles, another complex phenomena occur due to the fluid-solid and solid-solid interactions.

The variation of friction coefficient $f$ with $R e$ for different values of $\phi_{b}$ for the helical and chaotic flow configurations is shown in figure 15. As shown in this 

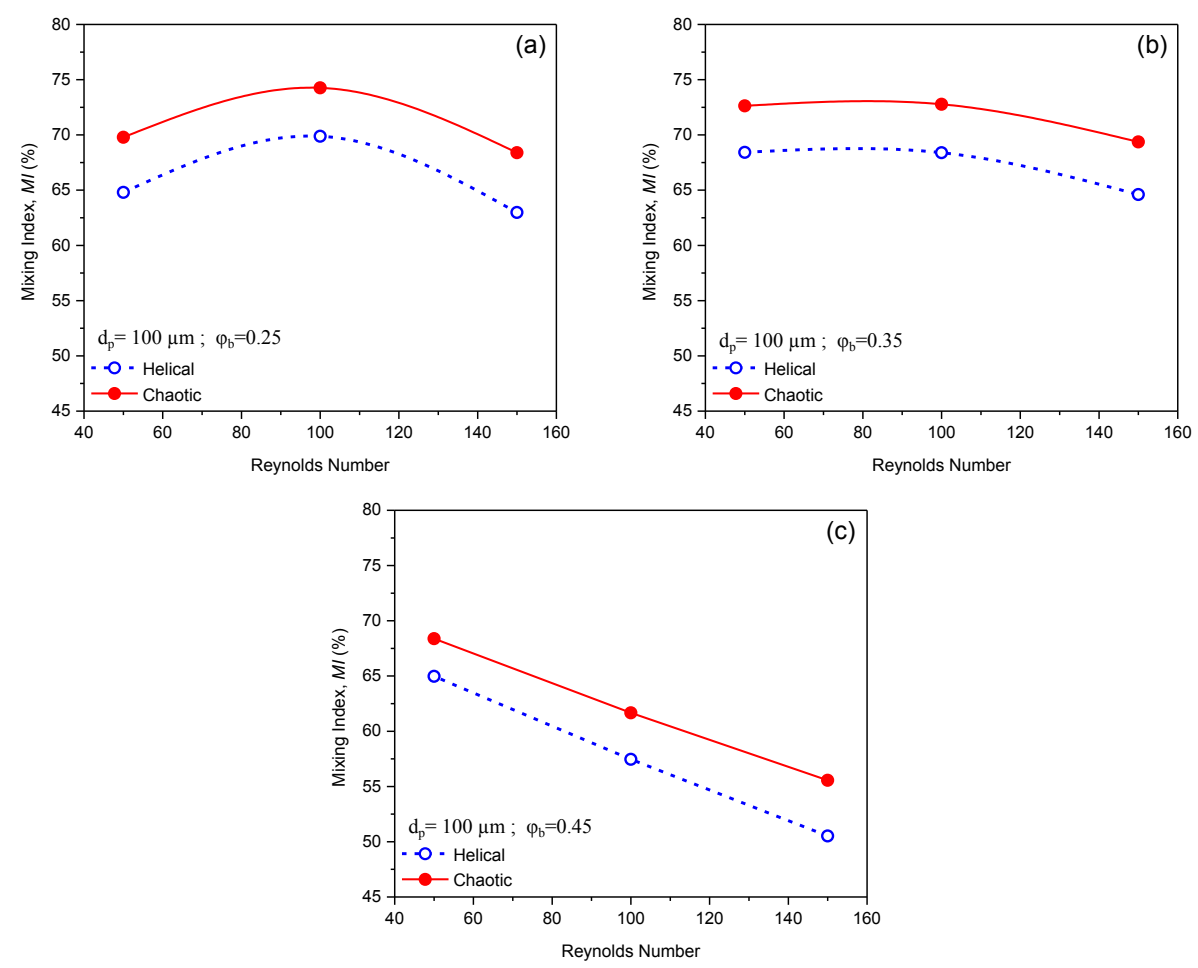

Figure 14: Mixing index variation versus Reynolds number for different values of concentration (a) $\phi_{b}=0.25$, (b) $\phi_{b}=0.35$ and $\phi_{b}=0.45$ with same $d_{p}=100 \mu \mathrm{m}$.

figure, the friction coefficient decreases with the Reynolds number according to a power law decay with an average value around -0.8. Exact correlations will be given in next sections. Moreover, almost no significant difference is observed between the helical and chaotic mixers which is in accordance to previous data for liquid flows [34]. In fact, the friction factor in the chaotic configuration is only $2 \%$ greater than that in the helical pipe flow. Moreover, increasing the particles concentration lead to a decrease in the friction coefficient which might be confusing for the reader, but this can be explained by our definition for the suspension friction coefficient (equation 18) which differs from the classical friction factor definition for pure fluids (we mean through a suspension velocity $U_{i n}\left(\phi_{b}\right)$ and not a pure fluid velocity). In fact, the present friction coefficient 
was defined based on a suspension effective velocity at the straight pipe's inlet $U_{\text {in }}\left(\phi_{\text {bulk }}\right)$, that increases as $\phi$ increases to retain same $R e_{S}$ values at different $\phi$ values. So the present $U_{i n}\left(\phi_{b u l k}\right)$ is inversely proportional to the friction coefficient defined in equation 18 which explains why $f$ decreases as $\phi$ increases.

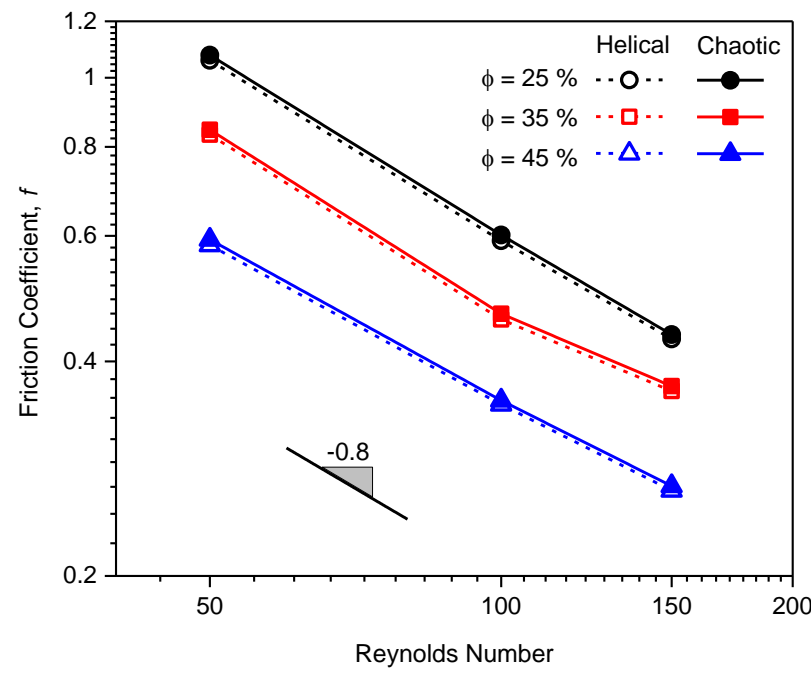

Figure 15: Friction factor variation versus Reynolds number for different values of concentration with same $d_{p}=100 \mu \mathrm{m}$.

To highlight the mixing performances of the helical and chaotic flow configurations, the mixing index is plotted against the pumping power factor $F$ in 16 for the different Reynolds numbers studied. As shown in this figure, at same pumping power, the chaotic mixer shows better performance than the helical mixer. Moreover, the best performances are obtained at lower Reynolds numbers. In fact, as observed in this figure, the pumping power increases with increasing Reynolds number accompanied by a slight decrease in the mixing index.

\subsection{Effect of volume fraction of particles}

In this section, we discuss the effect of varying the volume fraction of particles on the mixing performance in the helical coiled and chaotic twisted pipe 


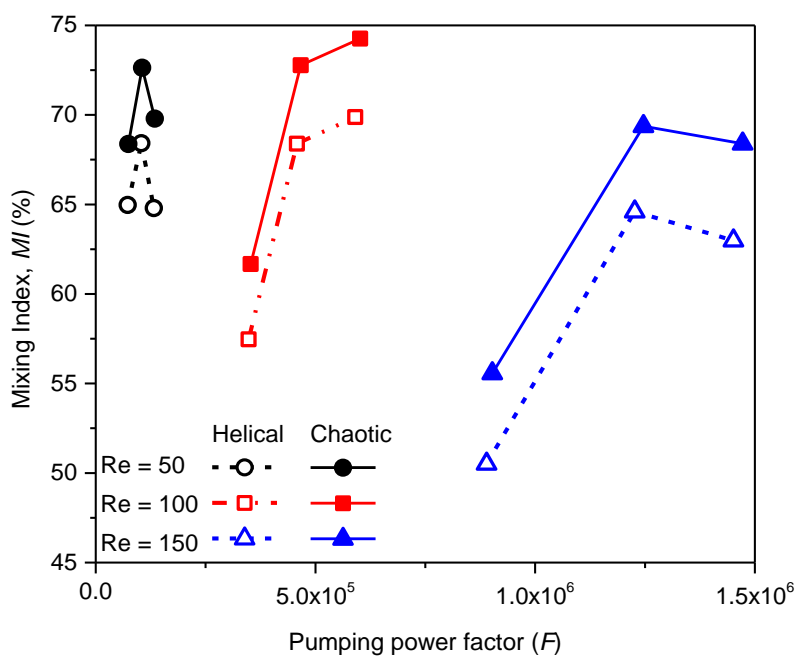

Figure 16: Pumping power factor for different values of Reynolds numbers and concentration: $d_{p}=100 \mu m$.

flows. Figure 17 shows the variation of $M I$ versus $\phi_{b}$ for different values of $R e$. As observed in this figure, the mixing index seems unchangeable with $\phi_{b}$ in the range between 0.25 and 0.35 for both configurations. Then the mixing index decreases when $\phi_{b}$ increases to its maximum value of 0.45 . In fact, this could be explained by the fact that the suspension flow goes from diluted to concentrated regime where the particles dynamics and their interaction with the flow field becomes different. These two different regimes were manifested through previous rheological measurements of the suspension such as the particles normal stress differences (see $[8,25])$.

The variation of the friction coefficient versus $\phi_{b}$ is shown in figure 18. As shown in this figure, $f$ shows a decrease with increasing $\phi_{b}$ with similar power law decay of -0.8 as observed for the variation of $f$ versus the Reynolds number. Similar conclusions could be extracted here as for the variation of $f$ versus $R e$ where the chaotic and helical flow configurations show similar friction losses.

Another important issue is representing the variation of the mixing index 

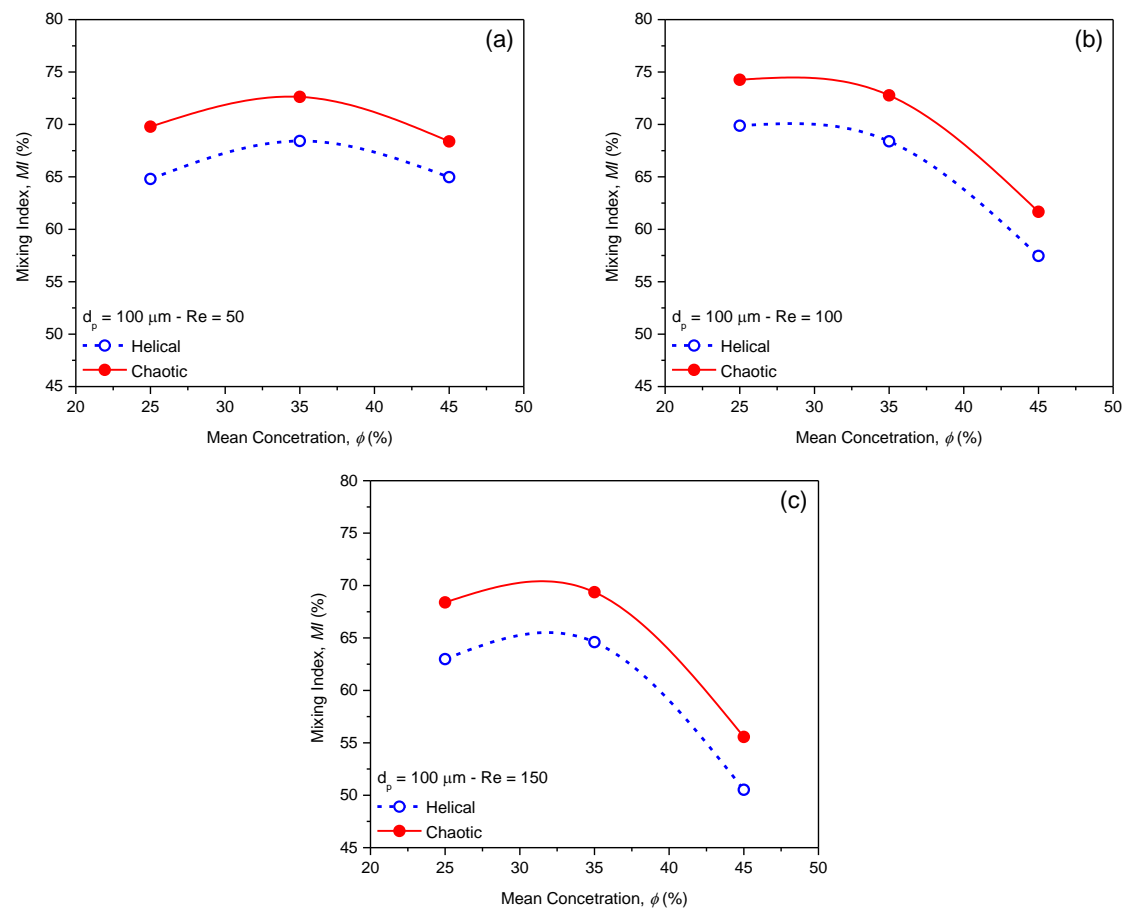

Figure 17: Mixing index variation versus concentration for different values of Reynolds numbers (a) $R e=50$, (b) $R e=100$ and $R e=150$ with same $d_{p}=100 \mu \mathrm{m}$.

versus the pumping power factor $F$ for different values of $\phi_{b}$ as shown in figure 19. From this figure it could observed that at the lowest values of $\phi_{b}$, the mixing index is almost constant while varying the pumping power with similar performances for both helical and chaotic configurations. Meanwhile, for $\phi_{b}=0.45$, the mixing index decreases by increasing the pumping power, which is somehow controversial, where the chaotic flow configuration show better performance than the helical mixer at same pumping power.

\subsection{Effect of particle diameter}

The effect of changing the particles diameter on the mixing performances and friction losses is discussed in this section. Figure 20 shows the variation of $M I$ versus $d_{p}$ for the following operating conditions: $\phi=0.35$ and $R e=100$. 


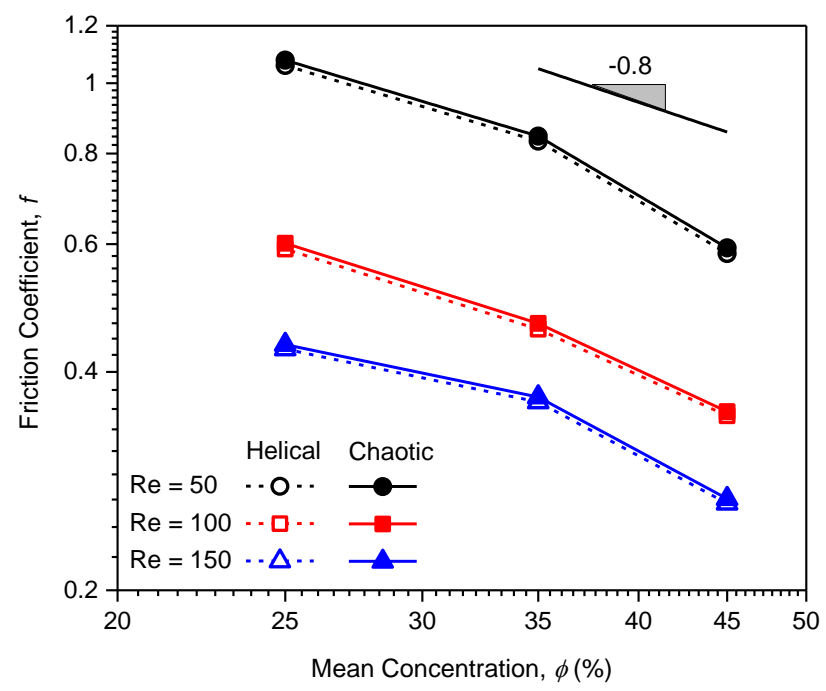

Figure 18: Friction factor variation versus Reynolds number for different values of concentration with same $d_{p}=100 \mu \mathrm{m}$.

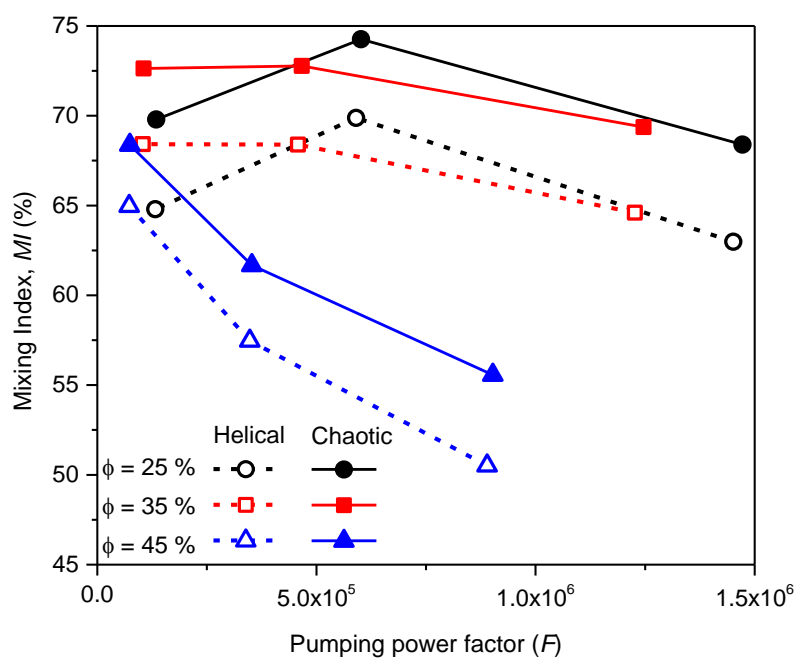

Figure 19: Pumping power factor for different values of Reynolds numbers and concentration: $d_{p}=100 \mu m$.

As shown in this figure, the mixing index increases drastically by increasing the particles diameter where it goes from around $15 \%$ for the lowest studied $d_{p}$ to around $70 \%$ at the highest particles diameter. Moreover, the chaotic 
configuration shows always better performances than the helical mixer for all the range of $d_{p}$.

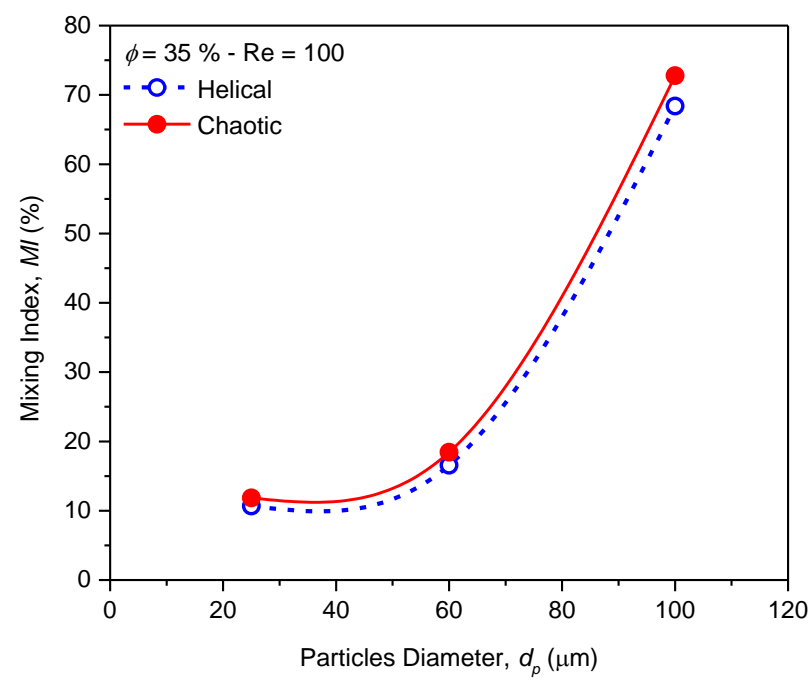

Figure 20: Mixing index variation versus particle diameter: $\phi=0.35-R e=100$.

Meanwhile, the friction coefficient represented in figure 21 shows very slight variation with the particles diameter. This means that $d_{p}$ has no significant effect of the friction losses, however it affects greatly the $M I$ of the static mixer user in the downstream of the straight pipe flow. In fact the independence of the friction factor $f$ on the particles diameter $d_{p}(21)$ can be explained, as stated earlier, due to our choice for the definition of the latter in equation 18, where $f$ depends on $R e_{S}$ which is $\eta_{S}$-dependent. The latter depends only on the volume fraction of particles $\phi$ as can be see in equation 5 .

\section{Conclusion and Perspectives}

The mixing enhancement quantification and mixing behavior in two static mixers (helical and chaotic pipes) for concentrated isothermal monodisperse neutrally buoyant non-colloidal suspension flows has been investigated numeri- 


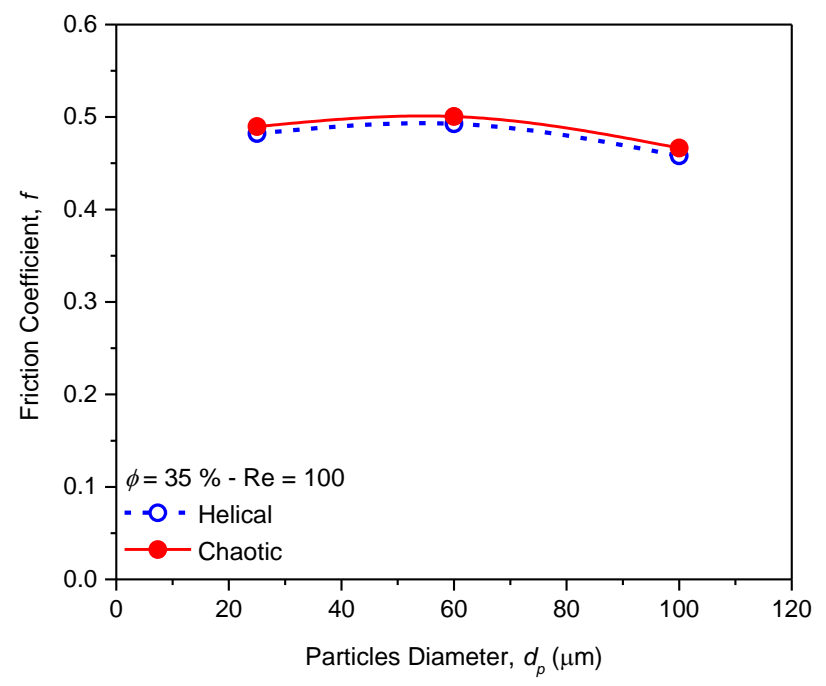

Figure 21: Friction factor variation versus particle diameter: $\phi=0.35-R e=100$.

cally through transient 3D CFD simulations (for the first time to authors knowledge).

The mixing enhancement is quantified for suspension effective Reynolds numbers varying between 50 and 150 (Dean numbers between 10 and 31), for sphere diameters between 25 and 400 micrometers and for particles volume fractions between 0.25 and 0.45 . The influences of $d_{p}$, Re and $\phi$ on the overall mixing enhancement and on pressure drop (friction factor) are quantified by applying a suspension flow in a chaotic and in a helical separate pipes as static mixers (each of circular cross section and of equal length).

It is shown that for all cases, the chaotic flow configuration has better mixing performances than the helical pipe flow with almost no significant increase in pressure losses. Good mixing quality is observed (better at larger particles) in both static mixers where the mixing index ranges between 10 and $75 \%$ relative to the initial unmixed particles leaving the straight pipe.

The present 3D modeling and simulation approach in CFD is very promis- 
ing for designing better future mixers for improved overall performances. This

is important for many engineering processes especially in the industrial and chemical engineering sectors where non-colloidal suspension flows are present.

Non-neutrally buoyant particles can be accounted for in the future by extending the presented numerical models and solvers. A challenge will be to solve numerical difficulties that may arise due to additional external forces and high density ratios between the particles and the surrounding liquid (i.e. gravitational forces, settling speeds, pressure-velocity coupling, etc).

Future experimental and numerical investigations are still required in order to understand more deeply the limits of the present numerical continuum modeling approach applied to mixing in concentrated non-Brownian suspension flows of mono- and poly- dispersed particles of different forms.

\section{References}

[1] S. Peker, S. Helvaci, Solid-Liquid Two Phase Flow, 1st Edition, Elsevier, Amsterdam, 2008.

[2] J. J. Derksen, Simulations of solid-liquid mass transfer in fixed and fluidized beds, Chemical Engineering Journal 255 (2014) 233-244.

[3] S. B. de Araujo, M. Carvalho, Sedimentation and marangoni stress in slot coating flow of particle suspension, J. Non-Newton. Fluid Mech. 247 (2017) $53-61$.

[4] R. Jukka, K. Johannes, The future of pharmaceutical manufacturing sciences, J. Pharm. Sci. 104 (11) (2018) 3612-3638.

[5] C. Dong, J. S. Zhang, K. Wang, G. S. Luo, Micromixing performance of nanoparticle suspensions in a micro-sieve dispersion reactor, Chemical Engineering Journal 253 (2014) 8-15. 
[6] L. N. Ejim, S. Yerdelen, T. McGlone, I. Onyemelukwe, B. Johnston, A. J. Florence, N. M. Reis, A factorial approach to understanding the effect of inner geometry of baffled meso-scale tubes on solids suspension and axial dispersion in continuous, oscillatory liquid-solid plug flows, Chemical Engineering Journal 308 (2017) 669-682.

[7] T. Dbouk, L. Lobry, E. Lemaire, F. Moukalled, Shear-induced particles migration; predictions from experimental determination of the particle stress tensor, J. Non-Newton. Fluid Mech. 198 (2013) 78-95.

[8] T. Dbouk, L. Lobry, E. Lemaire, Normal stresses in concentrated nonbrownian suspensions, J. Fluid Mech. 715 (2013) 239-272.

[9] T. Dbouk, A suspension balance direct-forcing immersed boundary model for wet granular flows over obstacles, J. Non-Newton. Fluid Mech. 230 (2016) 68-79.

[10] D. Leighton, A. Acrivos, Measurement of self-diffusion in concentrated suspensions of spheres, J. Fluid Mech. 177 (1987) 109-131.

[11] D. Leighton, A. Acrivos, The shear-induced migration of particles in concentrated suspensions, J. Fluid Mech. 181 (1987) 415-439.

[12] F. Gadala-Maria, A. Acrivos, Shear-induced structure in a concentrated suspension of solid spheres, J. Rheol. 24 (1980) 799.

[13] B. Chapman, Shear-induced migration phenomena in concentrated suspensions, Ph.D. thesis, University of Notre Dame, Indiana, USA (1990).

[14] I. Krieger, T. Dougherty, A mechanism for non-newtonian flow in suspensions of rigid spheres, Soc. Rheol. 3 (1959) 137.

[15] J. Richardson, W. Zaki, Sedimentation and fluidization:part i, Trans. Inst. Chem. Eng. 32 (1954) 35-47. 
[16] A. Chow, S. Sinton, J. Iwamiya, T. Stephens, Shear-induced migration in couette and parallel-plate viscometers: Nmr imaging and stress measurements, Phys. Fluids 6 (1994) 2561.

[17] F. Boyer, O. Pouliquen, E. Guazzelli, Dense suspensions in rotating-rod flows: Normal stresses and particle migration, J. Fluid Mech. 686 (2011) $5-25$.

[18] M. Lyon, L. Leal, An experimental study of the motion of concentrated suspensions in two-dimensional channel flow, part 1. monodisperse systems, J. Fluid Mech. 363 (1998) 25-56.

[19] J. Morris, F. Boulay, Curvilinear flows of non-colloidal suspensions: the role of normal stresses, J. Rheol. 43 (1999) 1213-1237.

[20] D. Merhi, E. Lemaire, G. Bossis, F. Moukalled, Particle migration in a concentrated suspension flowing between rotating parallel plates: investigation of diffusion flux coefficients, J. Rheol. 49 (2005) 1429.

[21] S. Gallier, E. Lemaire, L. Lobry, F. Peters, Effect of confinement in wallbounded non-colloidal suspensions, J. Fluid Mech. 799 (2016) 100-127.

[22] J. Morris, J. Brady, Pressure-driven flow of a suspension: Buoyancy effects, Int. J. Multiphase Flow 24 (1998) 105-130.

[23] T. Dbouk, Heat transfer and shear-induced migration in dense nonbrownian suspension flows: Modelling and simulation, J. Fluid Mech. 840 (2018) 432-452.

[24] E. Guazzelli, J. Morris, A Physical Introduction to Suspension Dynamics (Cambridge Texts in Applied Mathematics), Cambridge University Press, 2011. 
[25] E. Guazzelli, Rheology of dense suspensions of non colloidal particles, Powders and Grains EPJ Web of Conferences 140 (2017) 01001.

[26] F. Ramasomanana, M. Fahs, H. Baalousha, N. Barth, Z. Ahzi, An efficient ellam implementation for modeling solute transport in fractured porous media, Water Air and Soil Pollution 229 (2) (2018) .

[27] R. Nourgaliev, T. Dinh, T. Theofanous, D. Joseph, The lattice boltzmann equation method: theoretical interpretation, numerics and implications, International Journal of Multiphase Flow 29 (2003) 117-169.

[28] M. Coutinho, Guide to Dynamic Simulations of Rigid Bodies and Particle Systems (Simulation Foundations, Methods and Applications), Springer Publishing, 2013.

[29] K. Yeo, M. Maxey, Numerical simulations of concentrated suspensions of monodisperse particles in a Poiseuille flow, J. Fluid Mech. 682 (2011) 491518.

[30] D. Dherbcourt, S. Charton, F. Lamadie, S. Cazin, E. Climent, Experimental study of enhanced mixing induced by particles in Taylor-Couette flows, Chemical Engineering Research and Design 108 (2016) 109-117.

[31] M. Souzy, H. Lhuissier, E. Villermaux, B. Metzger, Stretching and mixing in sheared particulate suspensions, J. Fluid Mech. 812 (2017) 611-635.

[32] M. Souzy, X. Yin, E. Villermaux, C. Abid, B. Metzger, Super-diffusion in sheared suspensions, Phys. Fluids 27 (4) (2018) 041705.

[33] A. Mokrani, C. Castelain, H. Peerhossaini, The effects of chaotic advection on heat transfer, International Journal of Heat and Mass Transfer 40 (13) (1997) 3089-3104. 
[34] C. Castelain, D. Berger, P. Legentilhomme, A. Mokrani, H. Peerhossaini, Experimental and numerical characterisation of mixing in a steady spatially chaotic flow by means of residence time distribution measurements, International Journal of Heat and Mass Transfer 43 (19) (2000) 3687-3700.

[35] M. Jarrahi, C. Castelain, H. Peerhossaini, Mixing enhancement by pulsating chaotic advection, Chemical Engineering and Processing: Process Intensification 74 (2013) 1-13.

[36] B. Mondal, S. K. Mehta, P. K. Patowari, S. Pati, Numerical study of mixing in wavy micromixers: comparison between raccoon and serpentine mixer, Chemical Engineering and Processing - Process Intensification 136 (2019) $44-61$.

[37] B. Mondal, S. Pati, P. K. Patowari, Analysis of mixing performances in microchannel with obstacles of different aspect ratios, Proceedings of the Institution of Mechanical Engineers, Part E: Journal of Process Mechanical Engineering (2019) .

[38] C. Castelain, P. Legentilhomme, Residence time distribution of a purely viscous non-Newtonian fluid in helically coiled or spatially chaotic flows, Chemical Engineering Journal 120 (3) (2006) 181-191.

[39] C. Habchi, T. Lemenand, D. Della Valle, H. Peerhossaini, Liquid/liquid dispersion in a chaotic advection flow, International Journal of Multiphase Flow 35 (6) (2009) 485-497.

[40] C. Habchi, S. Ouarets, T. Lemenand, D. Della Valle, J. Bellettre, H. Peerhossaini, Influence of viscosity ratio on droplets formation in a chaotic advection flow, International Journal of Chemical Reactor Engineering 7 (A50) (2009) 1-14. 
[41] A. Tohidi, H. Ghaffari, H. Nasibi, A. S. Mujumdar, Heat transfer enhancement by combination of chaotic advection and nanofluids flow in helically coiled tube, Applied Thermal Engineering 86 (2015) 91-105.

[42] M. Bahiraei, N. Mazaheri, Second law analysis for flow of a nanofluid containing graphene-platinum nanoparticles in a minichannel enhanced with chaotic twisted perturbations, Chemical Engineering Research and Design 136 (2018) 230-241.

[43] W. R. Dean, LXXII. The stream-line motion of fluid in a curved pipe (second paper), The London, Edinburgh, and Dublin Philosophical Magazine and Journal of Science 5 (30) (1928) 673-695.

[44] S. Jones, O. Thomas, H. Aref, Chaotic advection by laminar flow in a twisted pipe, J. Fluid Mech. 209 (1989) 335-357.

[45] Y. Le Guer, H. Peerhossaini, Order breaking in Dean flow, Phys. Fluids 3 (5) (1991) 1029-1032.

[46] C. Habchi, J.-L. Harion, S. Russeil, D. Bougeard, F. Hachem, A. Elmarakbi, Chaotic mixing by longitudinal vorticity, Chemical Engineering Science 104 (2013) 439-450.

[47] C. Habchi, T. Lemenand, D. D. Valle, H. Peerhossaini, Alternating mixing tabs in multifunctional heat exchanger-reactor, Chemical Engineering and Processing: Process Intensification 49 (7) (2010) 653 - 661, part of special issue: Process Intensification on Intensified Transport by Complex Geometries. doi:https://doi.org/10.1016/j.cep.2009.07.003.

[48] R. Phillips, R. Armstrong, R. Brown, A. Graham, J. Abbott, A constitutive model for concentrated suspensions that accounts for shear-induced particle migration, Phys. Fluids 4 (1992) 30-40. 
[49] S. Yadav, M. M. Reddy, A. Singh, Shear-induced particle migration in three-dimensional bifurcation channel, International Journal of Multiphase Flow 76 (2015) 1-12.

[50] C. Habchi, J.-L. Harion, Residence time distribution and heat transfer in circular pipe fitted with longitudinal rectangular wings, International Journal of Heat and Mass Transfer 74 (2014) 13-24.

[51] P. Nott, J. Brady, Pressure-driven flow of suspensions: simulation and theory, J. Fluid Mech. 275 (1994) 157.

[52] R. Rebouças, I. Siqueira, P. de Souza Mendes, M. Carvalho, On the pressure-driven flow of suspensions: Particle migration in shear sensitive liquids, J. Non-Newton. Fluid Mech. 234 (2016) 178-187.

[53] M. Cross, Rheology of non-Newtonian fluids: a new flow equation for pseudoplastic systems, Journal of Colloid Interface Science 50 (1965) 417-437.

[54] S. Maron, P. Pierce, Application of free-eyring generalized flow theory to suspensions of spherical particles, Journal of Colloid Science 11 (1956) 162170.

[55] J. Stickel, R. Powell, Fluid mechanics and rheology of dense suspensions, Annu. Rev. Fluid Mech 37 (2005) 129-1149.

[56] C. Chang, R. Powell, Effect of particle size distributions on the rheology of concentrated bimodal suspensions, J. Rheology 38 (1) (1994) 85-98.

[57] R. Davis, A. Acrivos, Sedimentation of non-colloidal particles at low reynolds numbers, Annu. Rev. Fluid Mech. 17 (1985) 91-118.

[58] J. Ferziger, M. Peric, Computational Methods for Fluid Dynamics, Springer-Verlag Berlin and Heidelberg GmbH, 2002. 
[59] F. Moukalled, L. Mangani, M. Darwish, The Finite Volume Method in Computational Fluid Dynamics. An Advanced Introduction with OpenFOAM and Matlab, Springer International Publishing, 2015.

[60] OpenFOAM, Openfoam (Open Field Operation And Manipulation) open source CFD toolbox.

[61] I. Celik, U. Ghia, P. Roache, C. Freitas, H. Coleman, P. Raad, Procedure for estimation and reporting of uncertainty due to discretization in CFD applications, Journal of Fluids Engineering 130 (7) (2008) 078001.

[62] P. G. LaHaye, F. J. Neugebauer, R. K. Sakhuja, A generalized prediction of heat transfer surfaces, Journal of Heat Transfer 96 (4) (1974) 511-517. 


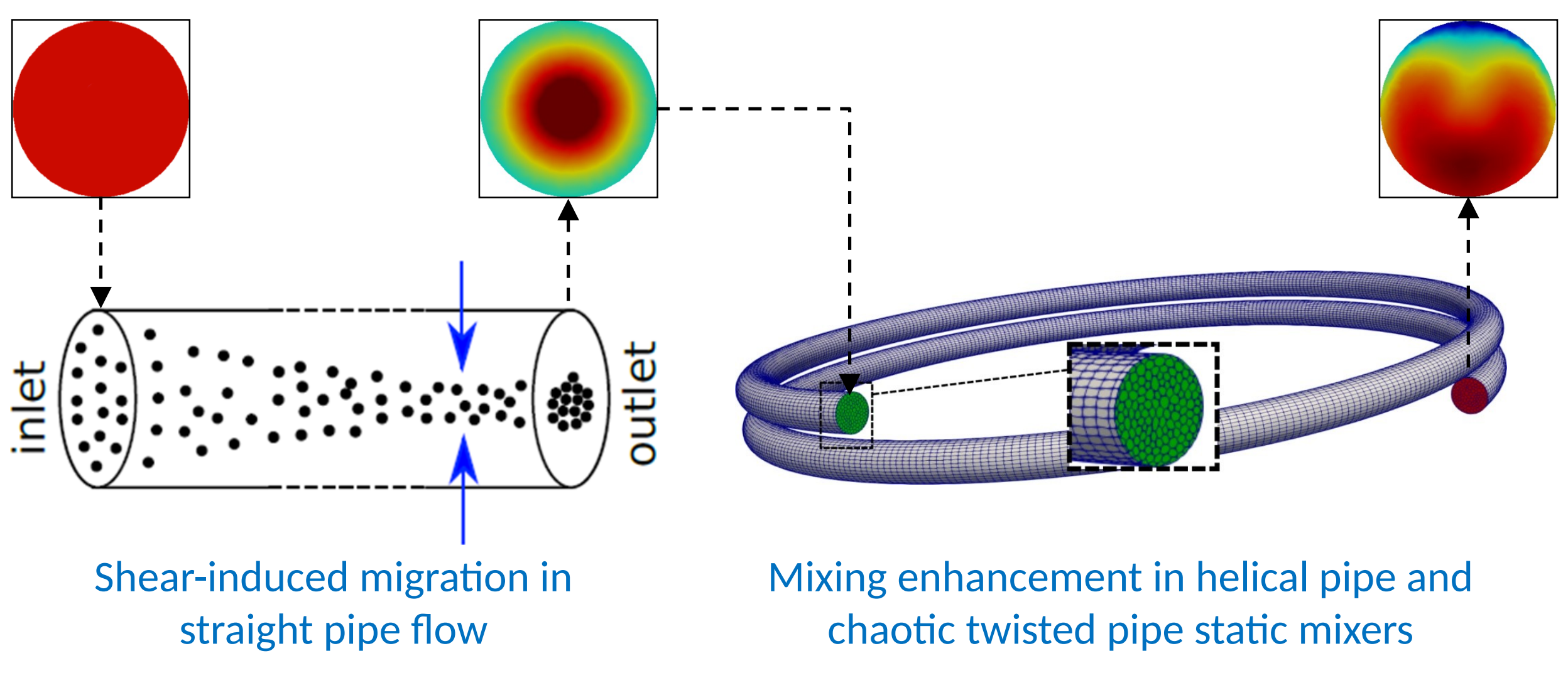

